\title{
Beatmungstherapie hilft Frauen mit Schlafapnoe nicht beim Überleben
}

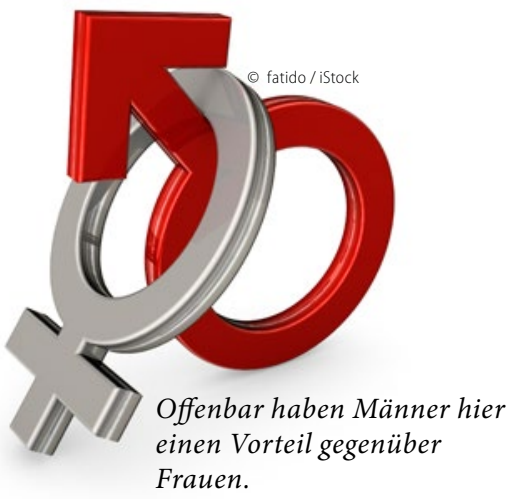

Fragestellung: Wie beeinflussen vorbestehende Komorbiditäten, Alter und Geschlecht den Effekt einer Continuous-Positive-Airway-Pressure-(CPAP-)Therapie bei obstruktiver Schlafapnoe (OSA) in Bezug auf die Mortalität?

Hintergrund: Die OSA ist assoziiert mit einer erhöhten Morbidität und Mortalität [1]. V.a. erhöht die OSA das Risiko für Hypertonie, Adipositas, Diabetes sowie kardio- und zerebrovaskuläre Erkrankungen [2]. Die CPAP-Therapie reduziert die OSA-assoziierte Morbidität und Mortalität [3]. Die dazu vorliegenden Studien beziehen sich aber im Wesentlichen auf Männer im mittleren Alter. Männer im höheren Alter sowie Frauen wurden bisher nicht genauer untersucht. Auch wurden bisher etwaige vorbestehende Komorbiditäten vor

Jennum P, Tønnesen P, Rikke I, Kjellberg J. All-cause mortality from obstructive sleep apnea in male and female patients with and without continuous positive airway pressure treatment: a registry study with 10 years of follow-up. Nature and Science of Sleep 2015; 7:43-50. der Diagnose einer OSA nicht erfasst bzw. ausgewertet.

Patienten und Methoden: Die Studie wurde unter Nutzung des dänischen nationalen Patienten-Registers, in der alle Erkrankungen bzw. Patienten mit Diagnose und The- rapie erfasst werden, durchgeführt. Es handelte sich somit um eine historische Kohortenstudie. Ausgewertet wurden die Daten von insgesamt 25389 Patienten mit der Diagnose einer OSA aus den Jahren 1999 bis 2009. U.a. wurde die sog. „All-Cause“-Mortalität aller Patienten ohne und mit CPAP-Therapie erfasst.

Ergebnisse: Unbehandelte OSA war insgesamt eng assoziiert mit einer niedrigeren Überlebensrate. Die Überlebensrate reduzierte sich mit zunehmendem Alter unabhängig vom Geschlecht. Frauen wiesen eine höhere Überlebensrate als Männer auf, und zwar unabhängig davon, ob sie auf eine CPAP-Therapie eingestellt waren oder nicht. Insgesamt reduzierte die CPAPTherapie aber die Mortalität um 38\%. Das Ausmaß der prädiagnostischen Morbidität hatte einen negativen Effekt auf die Überlebensrate. Die CPAP-Therapie hatte nicht nur einen positiven Effekt auf das Überleben bei Männern mittleren Alters, sondern auch bei einem Alter von 60 Jahren oder darüber. Es wurde aber keine signifikante Assoziation zwischen CPAP-Behandlung und Mortalität bei Frauen gefunden.

Schlussfolgerung: Die Autoren schlussfolgern, dass die CPAPTherapie bei OSA zwar die Mortalität bei Männern mittleren und höheren Alters signifikant senkt, aber nicht bei Frauen.

\section{- Kommentar von Prof. Dr. med. Kurt Rasche:}

\section{Viele Denkanstöße zum Thema „Frauen und Schlafapnoe“}

Obwohl die referierte Studie Limitationen aufweist, die die Autoren sehr ausführlich darstellen (historische Register-Analyse, keine Schweregrad-Bestimmung der OSA, fehlende Erfassung von "Confounding"-Faktoren wie BMI, keine zusätzlichen klinischen Daten), sind die Ergebnisse und Schlussfolgerungen der Arbeit sehr interessant und neu. Da bisher überwiegend Männer mittleren Alters in Studien zur Frage des CPAP-Effektes auf die Mortalität bei OSA untersucht wurden, eröffnet die vorliegende Arbeit Zugang zu der bisher wenig untersuchten Gruppe der Frauen mit OSA. Zum einen haben Frauen offensichtlich ein geringeres Risiko, an den Folgen der OSA zu sterben, zum anderen hat die CPAP-Therapie bei Frauen - zumindest nach den Daten dieser Studie - keinen signifikanten positiven Effekt auf die Überlebensrate. Die geringere Gesamtsterblichkeit von Frauen könnte - so argumentieren die Autoren - auf einen prämenopausalen kardio- und zerebrovaskulären hormonell bedingten Schutz zurückgeführt werden. Denkbar wäre auch eine gegenüber Männern anders strukturierte Weckbarkeit der Frauen, die dann wiederum möglicherweise zu geringeren kardiound zerebrovaskulären Auswirkungen der OSA führen könnte. Die Studie gibt daher viele Anregungen und Denkanstöße zum Thema „Frauen und Schlafapnoe”. Ganz sicher regt aber diese retrospektive und unkontrollierte Studie zu einer prospektiven und kontrollierten Studie zur Frage des CPAP-Effektes auf die Mortalität bei Frauen mit OSA an!

\section{Literatur}

1. Lavie P et al (2005) All-cause mortality in males with sleep apnoea syndrome: declining mortality rates with age. Eur Respir J 25:514-520.

2. Lavie P et al (2007) Mortality risk factors in sleep apnoea: a matched case-control study. J Sleep Res 16:128-134.

3. Marin JM et al (2005) Long-term cardivacular outcomes in men with OSAHS with and without treatment with CPAP: an observational study. Lancet; 365:1046-1053.

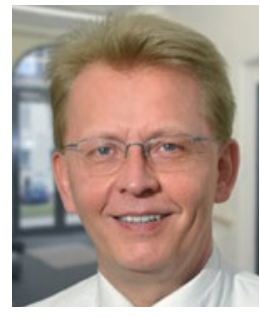

Prof. Dr. med. Kurt Rasche

HELIOS Klinikum Wuppertal

Bergisches Lungenzentrum

Klinikum der Universität Witten/Herdecke

Heusnerstr. 40, 42283 Wuppertal

kurt.rasche@helios-kliniken.de 